\title{
Video Article \\ Mindfulness in Motion (MIM): An Onsite Mindfulness Based Intervention (MBI) for Chronically High Stress Work Environments to Increase Resiliency and Work Engagement
}

\author{
Maryanna Klatt ${ }^{1}$, Beth Steinberg ${ }^{2}$, Anne-Marie Duchemin ${ }^{3}$ \\ 1 Department of Family Medicine, The Ohio State University College of Medicine \\ ${ }^{2}$ Critical Care Nursing, Wexner Medical Center \\ ${ }^{3}$ Department of Psychiatry, Stress, Trauma, and Resilience (STAR) Program, The Ohio State University College of Medicine
}

Correspondence to: Maryanna Klatt at Maryanna.Klatt@osumc.edu

URL: https://www.jove.com/video/52359

DOI: doi:10.3791/52359

Keywords: Behavior, Issue 101, Mindfulness, resiliency, work-engagement, stress-reduction, workplace, non-reactivity, Intensive-care, chronic stress, work environment

Date Published: 7/1/2015

Citation: Klatt, M., Steinberg, B., Duchemin, A.M. Mindfulness in Motion (MIM): An Onsite Mindfulness Based Intervention (MBI) for Chronically High Stress Work Environments to Increase Resiliency and Work Engagement. J. Vis. Exp. (101), e52359, doi:10.3791/52359 (2015).

\section{Abstract}

A pragmatic mindfulness intervention to benefit personnel working in chronically high-stress environments, delivered onsite during the workday, is timely and valuable to employee and employer alike. Mindfulness in Motion (MIM) is a Mindfulness Based Intervention (MBI) offered as a modified, less time intensive method (compared to Mindfulness-Based Stress Reduction), delivered onsite, during work, and intends to enable busy working adults to experience the benefits of mindfulness. It teaches mindful awareness principles, rehearses mindfulness as a group, emphasizes the use of gentle yoga stretches, and utilizes relaxing music in the background of both the group sessions and individual mindfulness practice. MIM is delivered in a group format, for $1 \mathrm{hr} /$ week $/ 8$ weeks. CDs and a DVD are provided to facilitate individual practice. The yoga movement is emphasized in the protocol to facilitate a quieting of the mind. The music is included for participants to associate the relaxed state experienced in the group session with their individual practice. To determine the intervention feasibility/efficacy we conducted a randomized wait-list control group in Intensive Care Units (ICUs). ICUs represent a high-stress work environment where personnel experience chronic exposure to catastrophic situations as they care for seriously injured/ill patients. Despite high levels of work-related stress, few interventions have been developed and delivered onsite for such environments. The intervention is delivered on site in the ICU, during work hours, with participants receiving time release to attend sessions. The intervention is well received with $97 \%$ retention rate. Work engagement and resiliency increase significantly in the intervention group, compared to the wait-list control group, while participant respiration rates decrease significantly pre-post in $6 / 8$ of the weekly sessions. Participants value institutional support, relaxing music, and the instructor as pivotal to program success. This provides evidence that MIM is feasible, well accepted, and can be effectively implemented in a chronically high-stress work environment.

\section{Video Link}

The video component of this article can be found at https://www.jove.com/video/52359/

\section{Introduction}

Mindfulness Based Stress Reduction (MBSR) ${ }^{1-3}$ is a stress reduction intervention that can be used to retrain the mind to change its usual responses to stressful situations. It has substantial research-based evidence for its efficacy in a variety of medical, social, educational, intercultural, and work-site settings ${ }^{4-6}$. MBSR teaches non-reactive awareness of one's affective response to external events and is presented as the key to changing one's internal experience of stress. Mindfulness is characterized by non-judgmental, sustained moment-to-moment awareness of physical sensations, perceptions, affective states, thoughts and imagery. MBSR is a time-intensive structured mind-body program (traditionally an 8 week, $26 \mathrm{hr}$ group intensive program) that utilizes mindfulness meditation and yoga postures to help manage a variety of adverse health issues, including stress. There are 35 years of empirical research illustrating the health benefits of this valuable approach. However, the required time commitment of traditional MBSR may negatively influence its applicability for personnel working in a chronically high stress work environment who may not have the time or energy to address their stress outside of work hours, but who could greatly utilize the benefits it affords.

The deleterious effects of chronically high stress work environments have been investigated ${ }^{7-8}$ but interventions to mediate this stress have been more recent ${ }^{9-13}$ as the impact of stress on personal physical and mental health has become more clearly established ${ }^{14-15}$. Effects of chronically high stress work environments also extend to the institutions that have to incur the costs of dealing with employee absenteeism and high turnover of expert personnel, such as highly trained nurses ${ }^{16}$. Interventions at the level of the organization (including mindfulness interventions) have been effective at reducing stress for personnel in various work environments, such as fire fighters, police officers, and prison guards ${ }^{17-19}$. But the actuality and severity of the situation for nurses in particular have been recognized by many as shown by the number of publications on the topic of nursing stress in recent years ${ }^{20-28}$. However, there are very few reports on pragmatic Mindfulness Based Interventions (MBIs) that 
have been successfully implemented during the workday, onsite, as part of an institutional initiative to combat stress and its impact in an attempt to transform organizational culture.

Mindfulness has been found useful as an intervention that increases attention ${ }^{29-30}$ and has been associated with changes in brain structure and function. For example the changes in gray matter brain density ${ }^{31}$ impacts cognition, while changes in the amygdala ${ }^{32}$ impact emotional reactivity. This may explain some of the positive benefits associated with stress reduction worksite interventions that teach non-reactivity for personnel who work in a chronic high stress work environment. Additionally, MBIs may impact the parasympathetic nervous system's tone, as the immune, autonomic, and endocrine systems all play a role in stress reactivity ${ }^{33}$, and have also been shown to decrease anxiety ${ }^{34}$. Each of these outcomes (especially increased ability to attend and increased parasympathetic tone) are valuable for personnel working in high stress work environments that are expected to provide compassionate care to those whom they serve while concurrently being highly focused on medical needs and changes. Although individuals who initiate their own personal stress reduction strategies may be able to lessen their individual experience of stress $^{17}$, systematic organization approaches are more effective. Workplace interventions can positively impact symptoms of stress (high blood pressure is one example) and demonstrate changes in psychological symptoms of stress ${ }^{35}$, and simply introducing stress reduction programming may shift the organizational climate, which has been shown to be an important factor for ICU nurses' intention to retain or leave their job ${ }^{36}$.

Turnover in high-stress jobs is of major concern for employers, including nursing turnover, due to the high cost to the institution ${ }^{16}$. Although there is variation in how studies calculate the cost of nurse turnover, estimates range approximately between $\$ 10,000-88,000$ per nurse, suggesting that nurse turnover is a concern, and costly for health care organizations ${ }^{16}$. In addition to this, research shows that patient satisfaction is less in hospitals with more dissatisfied nurses-a finding that may indicate a stressed, dissatisfied workforce may impact patient care ${ }^{37}$.

MIM is offered as a modified, less time intensive method to be delivered in the work place, and intends to enable busy working adults to experience the benefits of mindfulness. MIM teaches mindful awareness principles, rehearses mindfulness as a group, emphasizes the use of gentle yoga stretches, utilizes unique relaxing music in the background of group sessions/ individual practice, and requires daily individual mindfulness practice. The weekly session's content and structure follow that of the traditional MBSR, with an increased emphasis on bodily relaxation with the soft background music preceding the discussion of mindful awareness of cognitive habits. Participants receive three daily practice CDs (with 20 min practice tracks) and one yoga DVD with the background music and similar meditations as the ones practiced as a group, to be utilized for individual practice. These tools facilitate the ease of daily practice, increasing its feasibility. Development of this protocol is based on previous studies that suggest the efficacy of mindfulness interventions do not correlate with the length of time spent on the group didactic practice ${ }^{30,38-39}$, and yield similar results to the longer traditional MBSR. Non-reactive awareness of one's affective response to external events is presented as the key to changing one's internal experience of stress. MIM is specifically designed to be implemented in the workplace, during work hours, without the need to change clothes and can be done seated at a desk or standing. MIM is to be delivered onsite, during work hours for personnel exposed to stress and who are at high risk of developing burnout and could utilize the intervention to stay engaged and present in their work.

Mindfulness is the mental ability to enhance self-awareness of present internal and external experiences in a non-judgmental way. Mindfulness encourages people to become aware of how they are reacting to events as those events occur. It allows for a space of focused attention and awareness, in which the subject becomes able to watch his/her reaction to various events. Through attending to one's own reactions, in a nonjudgmental manner, one is able to reflect on the nature of the stressful event, and then able to evaluate if it is possible to reframe the event to lessen the perception of it being considered stressful ${ }^{1-4}$. MBls that occur in a group setting utilize group discussion of these realizations, which can result in community building amongst participants ${ }^{1,2}$. Such an experience may be a helpful worksite outcome. The self-reflection and awareness, and the shared experience of the emerging self-awareness, may contribute to a climate/culture change in a highly stressed work environment. Bishop ${ }^{40}$ generated a functional definition of mindfulness for researchers concerning the role and essential elements of an MBI. Two critical components were determined to be (1) self-regulation of attention and (2) the adoption of an orientation toward one's experiences in the present moment ${ }^{40}$. MIM, the onsite MBI protocol described in this manuscript was constructed to retain the essential elements of mindfulness, as it was conceived and has developed in traditional MBSR ${ }^{1-2}$, while adapting it in a pragmatic way for working adults. It utilizes the operational definition of mindfulness, yet, differs in the worksite location of the intervention, and the weekly time commitment of the group meeting and individual "homework" suggestion (Table 1).

\begin{tabular}{|c|c|c|c|c|c|}
\hline & Length of Intervention & Intervention Location & $\begin{array}{l}\text { Group Meeting } \\
\text { Duration }\end{array}$ & $\begin{array}{l}\text { Format of Group } \\
\text { Weekly Meeting }\end{array}$ & Daily Homework \\
\hline MBSR & 8 weeks & $\begin{array}{l}\text { Typically a location } \\
\text { other than a work site }\end{array}$ & $\begin{array}{l}2.5 \text { - } 3 \mathrm{hr} / \text { week, plus } \\
\text { one full day "retreat" }\end{array}$ & $\begin{array}{l}\text { Standard MBSR } \\
\text { program, Yoga } \\
\text { movement is often } \\
\text { done from the floor }\end{array}$ & $\begin{array}{l}45 \mathrm{~min}-1 \mathrm{hr} \text { of } \\
\text { mindfulness meditation }\end{array}$ \\
\hline MIM & 8 weeks & $\begin{array}{l}\text { Always on site location } \\
\text { (worksite for a work } \\
\text { study) }\end{array}$ & $\begin{array}{l}1 \mathrm{hr} / \text { week, plus one } 2 \\
\mathrm{hr} \text { "retreat" }\end{array}$ & $\begin{array}{l}\text { Modified MBI specific } \\
\text { for onsite delivery, } \\
\text { Yoga movement is } \\
\text { done standing or } \\
\text { seated, music in } \\
\text { background }\end{array}$ & $\begin{array}{l}20 \text { min of mindfulness } \\
\text { meditation via CD/ } \\
\text { DVD, music in } \\
\text { background }\end{array}$ \\
\hline
\end{tabular}

Table 1. Comparison of traditional MBSR as compared to MIM.

MIM is part of an organizational initiative to improve the resiliency and work engagement of employees and will be particularly important as it uses a "real-world" setting to address a critical problem experienced by many institutions dealing with chronically high stress work environments around the world.

The MIM protocol was developed by the first author to expose individuals who might benefit the most to mindfulness. As a trained yoga instructor (Yoga Alliance Certified) and attendee at an MBSR 9 day training for Health Professionals, she delivered MIM in this study, but has additionally designed a train-the-trainer program (for others with previous yoga/mindfulness training) in order to scale its delivery. Representative results of 
using MIM in a high stress environment (ICU) are presented in this manuscript, while biologic correlates to psychological outcomes associated with MIM will be forthcoming. This research provides an evidence-based approach for translation to practice at the level of the organization with the goal to improve work engagement in a specific population with potential application to employees working in other high stress environments, such as police officers, lawyers, fire fighters, etc.

\section{Protocol}

Institutional Review Board approval was obtained from The Ohio State University for this study and all participants signed an informed consent form.

\section{Characterize the High Stress Work Environment}

1. Meet with the managers and staff employees of the high stress environment separately from each other to characterize the impact of personal stress on the work environment. Assess various perspectives on the nature and sources of stress experienced by personnel. Ask both managers and staff employees for thoughts on causes and ways to modulate stress within the given environment.

2. Utilize all pre-existing quality improvement data concerning the nature of staff satisfaction, sick leave data, job turnover data etc. to determine level of personnel stress.

\section{Determine Appropriate Location/time/interest for MIM}

1. Communicate the research background on mindfulness and the intent to obtain institutional support for staff coverage necessary for participation.

2. Present findings of personnel stress assessment during staff meeting, determining time/location that would best work for MIM to be least disruptive for work flow.

\section{Obtain institutional support for MIM}

1. Work with administrative staff to allocate funding for coverage of staff attending MIM. Determine process for staff manager to solicit and schedule staff coverage for participants. Reserve location onsite for MIM.

2. Obtain written consent from interested employees so they fully understand the research, commitment involved in the intervention and understand that they can withdraw at any time, for any reason.

3. Decide upon the outcomes that would be clinically significant for the particular employees.

NOTE: For example, employees of ICU personnel showed a desire to become more resilient in spite of the daily stressors that they face in their day to day job and remain engaged in their important work.

\section{Baseline Assessments - 1 Week Prior to Intervention}

1. Measure resilience using the 10 -items version of the Connor-Davidson Resiliency Scale (CD-RISC) ${ }^{41} 1$ week before intervention to establish a baseline.

1. Ask participants to rate each of the 10 statements from 0 - 4 (not true at all, to true nearly all the time) indicating how often the statement applies to them.

2. Tally the total score by summing scores from each of the 10 statements.

NOTE: Resilience corresponds to the ability to maintain good functioning in face of stress or trauma. The 10-items version of the CDRISC has been validated with good reliability (alpha value of 0.85 ) and validity to differentiate individuals functioning well after adversity from those who are not ${ }^{42}$.

2. Measure work engagement using the 9-item Utrecht Work Engagement Scale (UWES) ${ }^{43} 1$ week before intervention to establish a baseline.

1. Ask participants to rate each of the 9 statements from $0-6$ (never to always) indicating how often the statement applies to them.

2. Tally the total score, as well as the 3 sub-scores for vigor, dedication and absorption.

NOTE: Higher scores indicate higher work engagement. Internal consistency of the scale is demonstrated with a Cronbach's alpha of 0.92 for the total score, 0.86 for vigor, 0.86 for dedication, and 0.79 for absorption subscales ${ }^{43}$.

\section{MIM}

Follow the same format during each $1 \mathrm{hr}$ weekly session of the 8 week program.

1. Begin each session by asking participants to count their respiration by placing their right hand on their chest and counting only inhales for 30 sec as timed by the instructor. Ask each participant to record their breath count on a log provided.

2. Play relaxing music in the background to set the climate for MIM.

3. State that the intent of the didactic/experiential sessions is to encourage the explicitly defined objective of the program: resiliency building and stress reduction through mindful awareness of habitual patterns of stress reactivity.

4. Each week, deliver a prompt for contemplation during the next hour and assure the participants that the response to the prompt is personal and silent. Invite the participants to choose to share responses, without any pressure to verbalize personal reflections. The prompts directly relate to each weekly theme. Ask the participants to reflect upon the following prompts: 


\begin{tabular}{|c|c|}
\hline Week & Prompt \\
\hline 1 & What physical or cognitive habits did you catch yourself doing during the last week? \\
\hline 2 & $\begin{array}{l}\text { What thoughts or worries keep you up at night? What thought, if you could put it out of your } \\
\text { mind, would allow you to sleep better? }\end{array}$ \\
\hline 3 & $\begin{array}{l}\text { What commonalities or differences do you see in your self-image versus how other people } \\
\text { see you? What image of self allows you to breathe easier and deeper? }\end{array}$ \\
\hline 4 & $\begin{array}{l}\text { Describe the most relaxed and satisfying meal you can remember. What factors made it so } \\
\text { enjoyable? }\end{array}$ \\
\hline 5 & $\begin{array}{l}\text { Are there any factors you can identify that were present during the times in your life when you } \\
\text { felt balanced? }\end{array}$ \\
\hline 6 & $\begin{array}{l}\text { What do you notice about the commonalities between the experiences you judge as } \\
\text { "pleasant?" What about the experiences you judge as "unpleasant?" }\end{array}$ \\
\hline 7 & $\begin{array}{l}\text { Is the "thing/person/situation" that is making you unhappy unchangeable? If so, how might } \\
\text { you be able to change your reaction to it or experience of that "thing/person/situation?" }\end{array}$ \\
\hline 8 & Describe a time at work that you felt grounded and empowered. \\
\hline
\end{tabular}

5. Deliver a 15 min Powerpoint presentation on topics including stress and work-related stress, theoretical material related to mindfulness, the somatic mind/body connection, relaxation, yoga, meditation, self-awareness, and bodily cues relating to emotional reactivity and the relation of these topics to the specific workplace stressors.

6. Following the prompt, lead the participants through a mind body relaxation relating to the weekly prompts:

1. Week 1: Discuss what types of communication/relational patterns participants notice in life, and how there are also bodily patterns of which people are often unaware. Ask the participants to cross their arms over their chest, then, to cross their arms the opposite way and notice whether or not this feels uncomfortable, or unusual, as it is different than their typical habit of which arm is typically on top. Ask the participants to relax their feet, their legs, drop their weight into the chair, relax shoulders down, relax the muscles of the face. Mindfully relax all the muscles of their body.

2. Week 2: Ask the participants to settle into their chairs. Instruct the participants to close their eyes and begin a body scan, thereby tensing and relaxing each part of the body. Ask the participants to bring attention to their feet, legs, buttocks, stomach, chest, shoulders, lips, open and close the lower jaw, relaxing it.

3. Week 3: Ask the participants to become aware of their breathing by concentrating on the rise and fall of their chest. For just a few minutes, allow the mind-chatter to become quiet. Instruct the participants to tense each part of the body (the lower body, the torso, the neck and shoulders, the head) and then release the tension as each body part relaxes.

4. Week 4: Pass out an orange and a small piece of dark chocolate. Instruct the participants to eat both of these with all five of their senses. For example, ensure that the participants hear the orange peel come off the orange, the wrapper off the chocolate. Direct the subjects to taste each of these 2 food items, smell each the orange and the chocolate, notice the difference and to notice the surface of the chocolate as opposed to the surface of the orange. Instruct the participants to take 3 min in silence to eat each of these 2 food items, noticing the experience, and the potential enhanced enjoyment of food, involved in mindful eating.

5. Week 5: Lead participants through yoga poses appropriate for the worksite, that can be performed without changing clothes or taking off one's shoes ${ }^{38}$ (balancing poses such as tree pose, eagle pose and chair pose). Ask the participants to notice the factors that are helpful to physically balance their body in these poses.

6. Week 6: Guide the participants through a self-massage as an example of a pleasurable break from stress. Instruct the participants to use all ten finger tips to begin massaging their head and scalp, moving down the back of the neck. Use the left hand to massage the right side of the neck, down the upper arm, to the elbow, ending at the palm of the hand and the fingers. Repeat using the right hand to massage the left side of the body.

7. Week 7: Ask participants to bring to mind the list of stressful items present in life. Instruct the participants to stand up. From a standing position, ask the participants to shift their weight to the right foot to represent the factors(e.g., people, situations, etc.) in their lives that cannot be changed, while tipping their torso to the left to represent the factors that are stressful yet are in part amendable to change. Ask participants to recognize that we all have both unchangeable and modifiable features present in our lives. Then ask the participants to rotate their shoulders in circles, followed by arms and hands moving in circles, ending by shaking out hands and participants return to seated position.

8. Week 8: Display an image of a mountain for the group. Ask the participants to imagine themselves as a solid mountain, with all the situations in life circling around the mountain while the mountain stays still. Imagine the body as an unmovable mountain.

7. End each session by asking each participant to count their respirations for $30 \mathrm{sec}$ and record their individual end-of-weekly-session breath count in the log provided. Assign the homework for the next week as follows.

1. Week 1: Ask the participants to notice each day the habits displayed - mental, physical, reactive, interactive. Instruct them to listen to a $\mathrm{CD}$ that has relaxing music in the background to reinforce the habit of relaxing for 20 min a day at least 5 days this week.

2. Ask the participants to notice what comes to mind when awake at night, or unable to fall asleep. Instruct them to listen to a CD that has relaxing music in the background that encourages falling asleep for $20 \mathrm{~min}$ a day at least 5 days this week.

3. Week 3: Ask the participants to notice breathing patterns this week in correlation with emotional states, and how breathing differently can change the emotional reactivity to a situation. Instruct them to listen to a CD that has relaxing music in the background that reinforces diaphragmatic breathing exercises that were practiced in the group session for 20 min a day at least 5 days this week.

4. Week 4: Ask the participants to notice where, when, and with whom meals are eaten, and the correlation with the enjoyment that is derived from eating food in a particular setting (for example in the car versus in one's dining room). Instruct them to practice mindful eating during one meal a day at least 5 days this week to reinforce the pleasure that can be derived from eating mindfully. 
5. Week 5: Ask the participants to notice on a daily basis when the feeling of balance is elicited by various situations or people. Instruct them to listen to a CD that has relaxing music in the background and that includes balancing yoga poses included for 20 min a day at least 5 days this week.

6. Week 6: Ask the participants to notice pleasurable versus uncomfortable sensations that occur daily and what or whom these experiences may be correlated with. Instruct them to listen to a CD that has relaxing music in the background that encourages pleasurable experiences 20 min a day at least 5 days this week.

7. Week 7: Ask the participants to notice which situations may have an element that can be changed to make the situation itself less stressful. Instruct them to listen to a CD that has relaxing music in the background that encourages flexibility to reinforce the content presented in the group session for 20 min a day at least 5 days this week.

8. Week 8: Ask the participants to notice situations at work (frustrations, successes) that are comparable to passing conditions that will not move a solid mountain (the participant). Instruct them to listen to a CD that has relaxing music in the background that encourages staying grounded for 20 min a day at least 5 days this week.

\section{Evaluation of MIM Delivered Onsite}

1. One week after the last session, ask the participants to rate the various component parts of the weekly sessions. Additionally, ask them to fill out a post intervention survey to measure resilience and engagement (see step 4.2 and 4.3 ).

2. Using a spreadsheet program evaluate the results obtained for differences in respiration by plotting the scores for that weekly session of the breath count (the average of all participants) at the beginning and end of each weekly session average breath count.

3. In a similar fashion, using a spreadsheet program, assess the resiliency and work engagement scores (total and sub-scales scores) for each group. Assess the changes between baseline and 2 month values in each study arm (MIM and group randomized to waitlist) using the paired t-test, consider $\mathrm{P}<0.05$ as significant.

\section{Representative Results}

Breath counts were self-measured by participants $(n=34)$ at the beginning and the end of each weekly session. Averaging the pool of participant breath counts revealed a decrease in the breath count between the beginning and the end of each weekly session. Individual participants associate the somatic experience of relaxation/clarity with the quantification of their own breath count difference; comparing respiration rates before each weekly session to those obtained at the end of each weekly session. Figure 1 shows the decrease in the 30 sec breath count as statistically significant for most time points (6/8 of the weekly session averages). There was also a decrease in the average number of breaths at the beginning of the 8 week program, between the 1st session (week 1) and the last session (week 8). Figure 1 shows participant's average pre-session respiration rate as 8.17 breaths/30 sec at week 1 , as compared to $6.61 / 30 \mathrm{sec}$ at week 8 . A similar pattern was seen in the average number of breaths at the end of the session from $5.03 / 30 \mathrm{sec}$ at week 1 to $3.63 / 30 \mathrm{sec}$ at week 8 ; however these were not statistically significant.

An associated goal of this intervention was that the personnel under chronic high stress in their work environment would increase the ability to remain resilient in the face of the stressful environment. Figure 2 shows that on the Connor-Davidson Resiliency Scale ${ }^{41}$ the scores were significantly different between pre- and post- 8 week intervention for the MIM group $(112.9 \%, p=0.0230, t=2.397)$, with no change in the waitlist control group $(101.7 \%, p=0.7330, t=0.3345)$, indicating that this intervention increased resiliency.

The organization that committed to funding this initiative was interested in creating a more resilient work force in spite of the stressful nature of the job responsibilities. Figure 3 shows that in the ICU personnel, the total work engagement average score indicated via the Utrecht Work Engagement Scale ${ }^{43}$ was significantly increased between pre- and post- 8-week intervention in the MIM group $(111.3 \%, p=0.0128, t=2.802)$. There was no change in the wait-list control group $(104.5 \%, p=0.1349, t=1.587)$. Most of the change was induced by an increase in the vigor subscale. Vigor average score increased between pre and post-8 week intervention in the MIM group $(117.9 \%, p=0.0331, t=2.331)$ with no change in the wait-list group $(101.8 \%, p=0.5624, t=0.5934)$. Dedication and absorption subscales scores were not significantly different in any groups.

Given that the focus of this study was to develop a MBI that could pragmatically be delivered onsite, it was important to determine which component parts of the intervention mattered to the participants. Table 2 shows participant rating of the component parts of MIM. On a scale from 1 to 10 (with 1 being the least valuable, and 10 being the most valuable), participants rated the usefulness of the various aspects of MIM. The highest scores were for the trained instructor $(M=8.67 \pm 0.71)$, music $(M=8.1 \pm 2.2)$, breathing awareness $(M=7.9 \pm 1.3)$, meditation $(M=$ $7.5 \pm 1.5)$, yoga stretches $(M=7.1 \pm 2.0)$, mindful eating $(M=6.8 \pm 1.7)$. This was congruent with qualitative comments made by the participants during the 8 week program. The first author was the instructor in this study, but has developed an extensive train the trainer program that has been successfully delivered in other locations. Participants value the evidenced-based nature of MIM, and appreciated the combination of the relaxing music played in the background and pragmatic practice tools provided. Regarding the satisfaction with the environmental constraints, the highest satisfaction was with the intervention location being onsite $(7.8 \pm 1.8)$, weekly sessions $(7.7 \pm 1.3)$, and duration of the intervention $(6.5 \pm 2.7)$, while time of the weekly session had the lowest satisfaction rating $(4.8 \pm 3.4)$. This low score proved surprising as the timing of the weekly session was chosen based upon staff recommendation. The importance of institutional support had a very high score at $8.1 \pm 1.8$ as well as the importance of patient care coverage $(7.9 \pm 1.9)$. It was very important to the ICU personnel to know that their patients were well cared for while they attended MIM. 


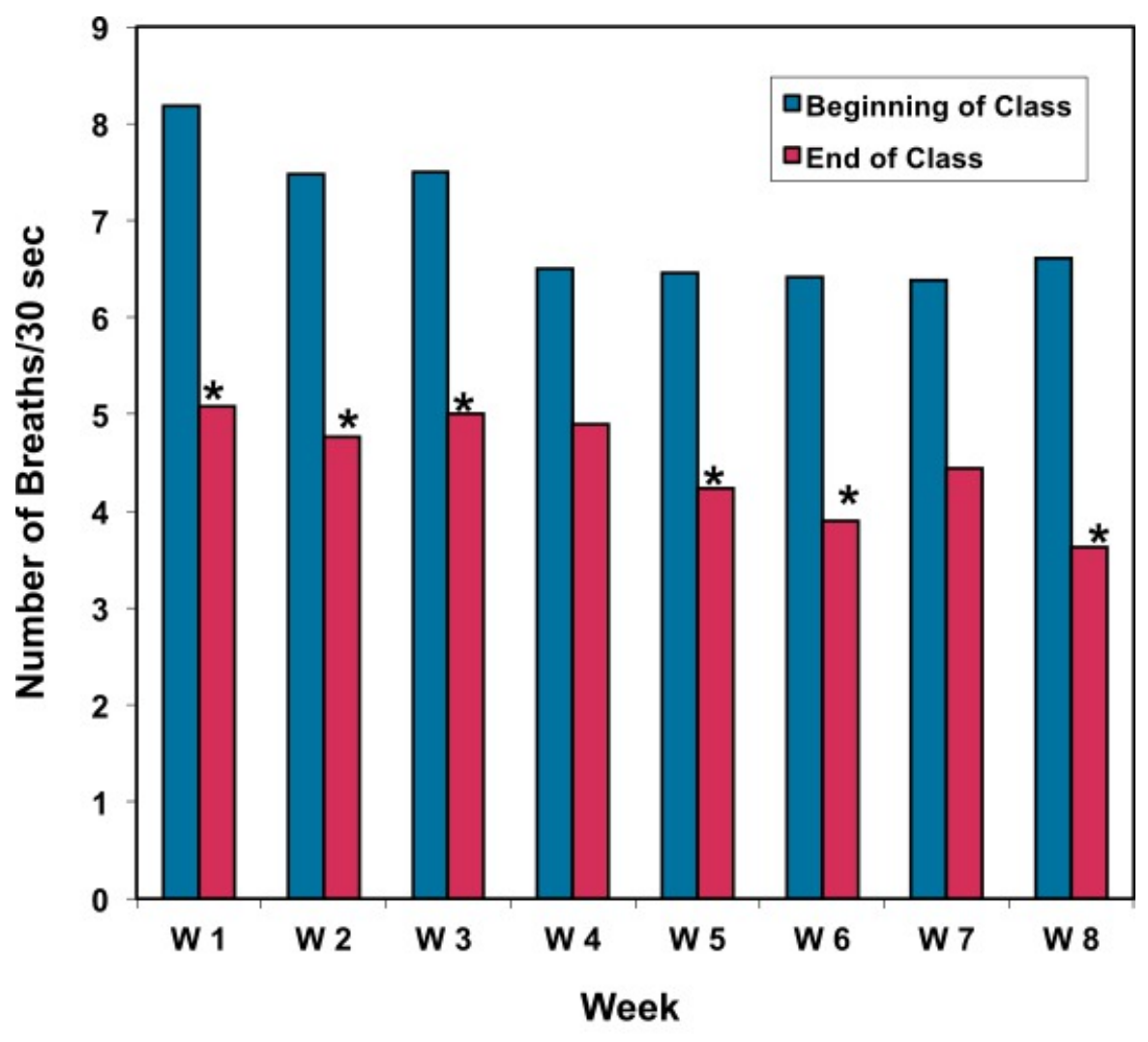

*Denotes significant difference between beginning and end of class $(p<0.05)$

Figure 1. Participant breath counts/30 sec pre to post weekly MIM session. There is a significant difference reflected in the pre-post breath counts in weeks $1-3,5-6$, and week 8 of the MBI intervention $(n=34)$. 


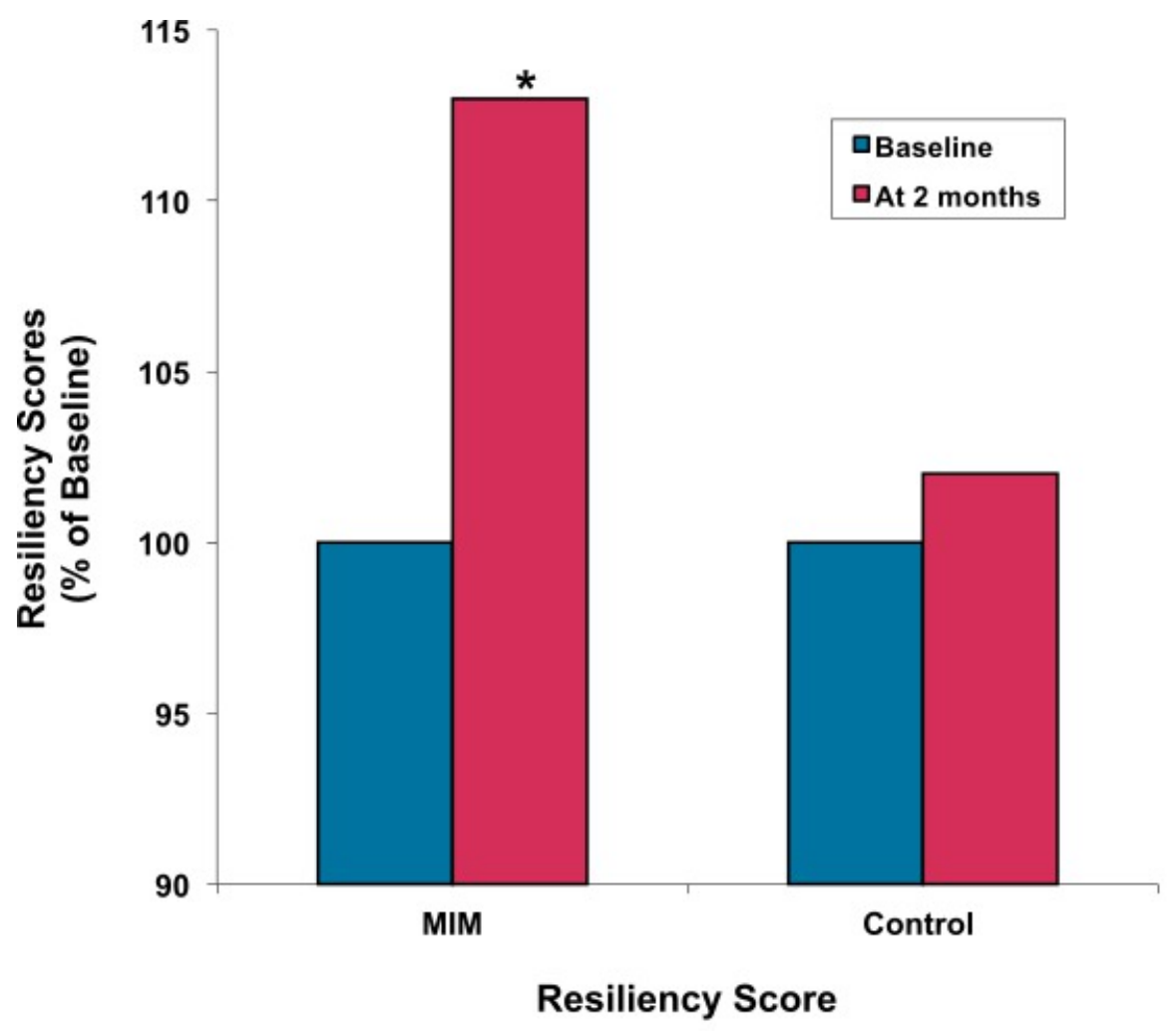

*Denotes significant difference between scores at baseline and 2 months $(p<0.05)$

Figure 2. Pre-post resiliency scores for the MIM intervention group compared to the wait-list control group. The MIM group improved significantly over the 8 weeks of the intervention in terms of resiliency skills $(n=34)$. 


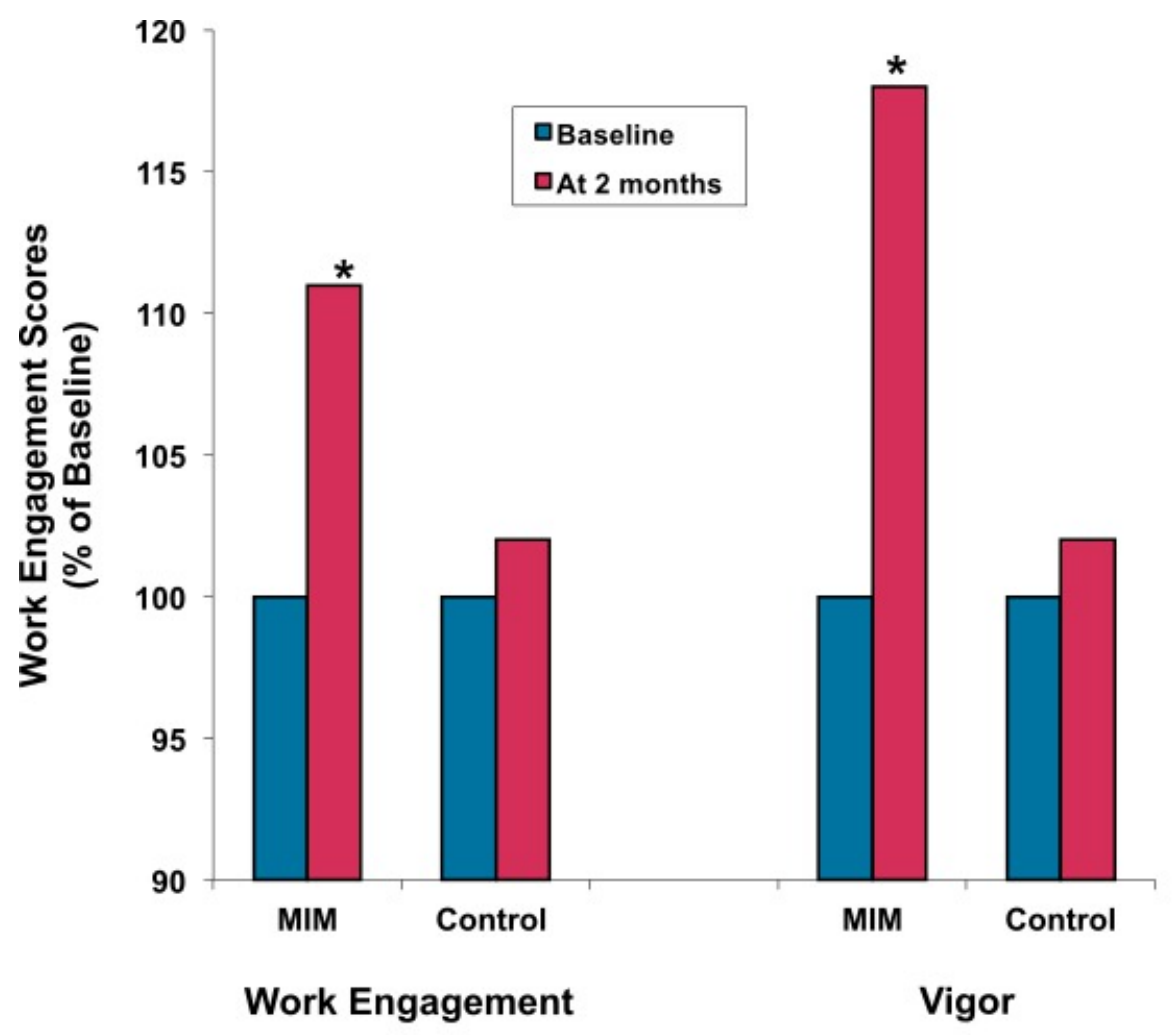

*Denotes significant difference between scores at baseline and 2 months $(p<0.05)$

Figure 3. Pre-post MIM work engagement scores. The MIM group significantly improved their sense of being engaged in their work over the 8 weeks of the MBI intervention $(n=34)$. There are three subscales on the Utrecht Work Engagement Scale: vigor, absorption, and dedication. Of these three subscales the MIM intervention group significantly improved in their sense of vigor towards their work, while dedication and absorption in work were not significantly different at the end of the 8 week program as compared with baseline. 


\begin{tabular}{|l|l|}
\hline MIM Program Components & Mean \pm SD \\
\hline Body scan & $6.69 \pm 1.81$ \\
\hline Yoga stretches & $7.06 \pm 2.01$ \\
\hline Breathing awareness & $7.94 \pm 1.34$ \\
\hline Meditation & $7.53 \pm 1.54$ \\
\hline Music & $8.06 \pm 2.26$ \\
\hline Mindful eating & $6.81 \pm 1.72$ \\
\hline Teaching handouts & $7.23 \pm 1.43$ \\
\hline CD & $8.00 \pm 1.06$ \\
\hline DVD & $7.92 \pm 1.02$ \\
\hline Weekly session & $7.75 \pm 1.29$ \\
\hline Location & $7.82 \pm 1.84$ \\
\hline Time of session & $4.76 \pm 3.41$ \\
\hline Duration (8 weeks) & $6.53 \pm 2.67$ \\
\hline Trained instructor & $8.67 \pm 0.71$ \\
\hline Group dynamics & $7.65 \pm 1.45$ \\
\hline Institutional support & $8.06 \pm 1.78$ \\
\hline Patient care coverage & $7.87 \pm 1.92$ \\
\hline
\end{tabular}

Table 2. Participant rating of MIM components. Rating on a 1 - 10 scale with 10 being most valued $(n=34)$. The institutional support was highly valued by the participants as this is what enabled them to receive time release to attend MIM. Other shift nurses were paid to come in an hour before their normal start time so that the MBI participant's patients were cared for by experienced nurses. The music that was played in the background of both the group sessions and on each of the individual CD tracks for individual practice was also very highly rated. Participants emphasized hearing the music played in the background of their individual practice reminded them of how relaxed they felt at the end of each weekly group session. The onsite location of the weekly sessions was also highly valued by participants.

\section{Discussion}

The rationale for developing the MIM protocol was to deliver a pragmatic onsite MBI to introduce mindfulness to working adults, within the context of a chronically high stress work environment, who may not have the time, nor inclination, to sign up for a mindfulness course outside of work hours, yet could significantly benefit from the resources it provides. Mindfulness research has consistently validated the contribution that mindfulness can offer towards emotion regulation ${ }^{20}$ and the value of remaining present, rather than worrying about the past, or thinking about the future ${ }^{4}$. For personnel, working in a high stress environment that concurrently demands high attention to detail, and extreme presence of mind to changing circumstances (such as an ICU patient's health status), the resources of emotion regulation and resiliency seem critical. But very few empirical studies have examined the benefit of providing mindfulness interventions in the workplace.

Yet workplace delivery, if institutional support can be garnered, is prudent as typical adults spend increasing amounts of time on the job. Attaining institutional support for both the cost of the intervention, and for the time coverage provided for the employees to attend the intervention, is critical to the success of this protocol. Participants need to feel supported by the organization, and providing the opportunity for an employee to attend a stress reduction intervention at their job is one way to accomplish this. Another critical feature is obtaining quality data pre and post intervention to demonstrate that the organizational investment was worthwhile. Subsequently presenting this data to policy and decision makers will ensure continued success of the intervention within various other units of the organization.

Modifications, easily made to MIM are critical as they show the employees that the intervention addresses their specific stresses and concerns, applied to their unique environmental stresses. Shortening the length of the weekly meetings from the traditional $2.5-3 \mathrm{hr}$ MBSR weekly meeting, to $1 \mathrm{hr}$ at shift change, was a seminal modification for this specific population of ICU personnel. Efforts were made before the intervention took place to accommodate the greatest amount of potential participants before choosing the time/location of the weekly one hour group meeting. Recent research that reviewed empirical mindfulness research interventions with nurses, notes that of the 13 studies included, only 2 studies were modified in terms of intervention length, and that most mindfulness interventions to date follow the traditional MBSR format and duration ${ }^{25}$. This provides the motivation to detail an onsite MBI that proves effective in increasing resilience and work engagement, specifically vigor in one's work, so that other professions, and work sites can adapt it for their own needs.

A limitation of this protocol, delivered on-site at the worksite is the absolute necessity of institutional support. Without replacement nursing personnel being paid to cover the hour of participation in the intervention, study participants would have had to attend on their own family/ personal time, lessening the sense that the organization is committed to the health and wellness of their employees. Another limitation is that a conveniently located conference room needs to be available for the intervention to occur.

The protocol described herein is highly adaptable to other environments. This research successfully illustrates the feasibility and effectiveness of implementation of MIM within the confines of the unique constraints of emergency situations, shift work, personnel coverage, space limitations, and many institutional regulations characteristics of this environment. Assessment of the specific stresses within the ICU proved to be a critical 
step in the protocol as the participants were appreciative of learning specific tools to interrupt common habitual reactions to stress, revealed to the ICU personnel during MIM. The stress assessment phase of information gathering enabled this to occur. Teaching specifics on how to cultivate more adaptive ways of responding to these stressors becomes a critical piece of the didactic portion of MIM. The breath counts conducted at the beginning and end of each weekly session gives participants a way to quantify a physical index of their stress level as they become increasingly aware that respiration rates are both reflective of stress levels, and can serve as an avenue that can be utilized to calm one self. Institutional support plays a critical role throughout the development and implementation of an onsite $\mathrm{MBI}$, as time release and adequate coverage is a necessary feature for the ICU personnel to be present to the weekly group meeting. Each feature of the MIM protocol described is at once important, yet flexible, able to meet the needs of other chronically high stressed work environments.

Additional research studies utilizing MIM, and development of other MBIs appropriate for the workplace will help to further policy change enabling mindfulness programs to be delivered routinely ${ }^{25}$. The largest study thus far of mindfulness in the workplace ${ }^{13}$ compared a MBI, delivered both online and in person. The control conditions included a therapeutic yoga group and also a group that did not receive any intervention. The 239 employees that were participants in the mind-body interventions showed significantly greater improvements in their perceived stress, the quality of their sleep, and heart rate variability. These are important findings as it indicates that the mind/body interventions helped the employees be more flexible in their stress responses. There were no differences between employee outcomes for those who attended in-person or online ${ }^{13}$. Some work environments that have unique constraints, (shift work in particular), may be best suited for an online delivery model to accommodate fluctuating work schedules. Thus, intervention length and location of $\mathrm{MBI}$ delivery are critical factors to consider for specific work environments, as well as method of delivery, e.g., in-person versus online delivery. Development of a pragmatic MBI that proves effective in a high stress, low control environment, such as the ICU, can effectively be delivered in other high stress work environments that require personnel to display high attention to detail, such as fire fighters, or police officers. The significance of implementing a pragmatic protocol that includes a holistic mind/body approach that considers modifications appropriate for the specific environment (intervention length /location) allows participation from employees that may never elect to participate in a longer, less convenient stress-reduction intervention. Additionally, increasing resilience and work engagement, and especially vigor in a population of personnel working in a high stress environment not only improves the lives of individuals, but potentially the lives that they touch through their work.

\section{Disclosures}

Subsequent to the completion of this research conducted at the Ohio State University, Dr. Klatt has served as a consultant to Mindful Management, LLC, to whom The Ohio State University has licensed the rights of the individual practice CD/DVD, and all other authors have nothing to disclose.

\section{Acknowledgements}

The authors acknowledge the following entities at the Ohio State University: Stress, Trauma, and Resilience (STAR) Program, Health System Administration, Critical Care Nursing, and the Faculty Associates Program through the Women's Place, for their critical institutional and financial contributions to this project.

\section{References}

1. Kabat-Zinn, J. An outpatient program in behavioral medicine for chronic pain patients based on the practice of mindfulness meditation: theoretical considerations and preliminary results. Gen Hosp Psychiatry. 4, (1), 33-47 (1982).

2. Kabat-Zinn, J., Hanh, T. N. Full catastrophe living: Using the wisdom of your body and mind to face stress. Delacourt (1990).

3. Ludwig, D. S., Kabat-Zinn, J. Mindfulness in medicine. JAMA. 300, (11), 1350-1352 (2008).

4. Brown, K. W., Ryan, R. M. The benefits of being present: mindfulness and its role in psychological well-being. J Pers Soc Psychol. 84, (4), 822-848 (2003).

5. Grossman, P., Niemann, L., Schmidt, S., Walach, H. Mindfulness-based stress reduction and health benefits. A meta-analysis. J Psychosom Res. 57, (1), 35-43 (2004).

6. Davidson, R. J., et al. Alterations in brain and immune function produced by mindfulness meditation. Psychosom Med. 65, (4), 564-570 (2003).

7. Johnson, J. V., Stewart, W., Hall, E. M., Fredlund, P., Theorell, T. Long-term psychosocial work environment and cardiovascular mortality among Swedish men. Am J Public Health. 86, (3), 324-331 (1996).

8. Juster, R. P., McEwen, B. S., Lupien, S. J. Allostatic load biomarkers of chronic stress and impact on health and cognition. Neurosci Biobehav Rev. 35, (1), 2-16 (2010).

9. Strijk, J. E., Proper, K. I., van Mechelen, W., van der Beek, A. J. Effectiveness of a worksite lifestyle intervention on vitality, work engagement, productivity, and sick leave: results of a randomized controlled trial. Scand J Work Environ Health. 39, (1), 66-75 (2013).

10. Chong, C. S., Tsunaka, M., Tsang, H. W., Chan, E. P., Cheung, W. M. Effects of yoga on stress management in healthy adults: A systematic review. Altern Ther Health Med. 17, (1), 32-38 (2011).

11. Dane, E., Brummel, B. J. Examining workplace mindfulness and its relations to job performance and turnover intention. Human Relations. 67, (1), 105-128 (2014).

12. Ho, L. A. Meditation, learning, organizational innovation and performance. Industrial Management, \& Data Systems. 111, (1), 113-131 (2011).

13. Wolever, R. Q., et al. Effective and viable mind-body stress reduction in the workplace: a randomized controlled trial. $J$ Occup Health Psychol. 17, (2), 246-258 (2012).

14. Kiecolt-Glaser, J. K., et al. Chronic stress and age-related increases in the proinflammatory cytokine IL-6. Proc Natl Acad Sci USA. 100, (15), 9090-9095 (2003).

15. McEwen, B. S. Central effects of stress hormones in health and disease: Understanding the protective and damaging effects of stress and stress mediators. Eur J Pharmacol. 583, (2), 174-185 (2008).

16. Li, Y., Jones, C. B. A literature review of nursing turnover costs. J Nurs Manag. 21, (3), 405-418 (2013). 
17. Oginska-Bulik, N. The role of personal and social resources in preventing adverse health outcomes in employees of uniformed professions. Int J Occup Med Environ Health. 18, (3), 233-240 (2005).

18. Chopko, B. A., Schwartz, R. C. The relation between mindfulness and posttraumatic growth: A study of first responders to trauma-inducing incidents. Journal of Mental Health Counseling. 31, (4), 363-376 (2009).

19. Williams, V., Ciarrochi, J., Patrick Deane, F. On being mindful, emotionally aware, and more resilient: Longitudinal pilot study of police recruits. Australian Psychologist. 45, (4), 274-282 (2010).

20. Hulsheger, U. R., Alberts, H. J., Feinholdt, A., Lang, J. W. Benefits of mindfulness at work: the role of mindfulness in emotion regulation, emotional exhaustion, and job satisfaction. J Appl Psychol. 98, (2), 310-325 (2013).

21. Shapiro, S. L., Astin, J. A., Bishop, S. R., Cordova, M. Mindfulness-based stress reduction for health care professionals: results from a randomized trial. International Journal of Stress Management. 12, (20), 164 (2005).

22. Pipe, T. B., et al. Nurse leader mindfulness meditation program for stress management: a randomized controlled trial. J Nurs Adm. 39, (3), 130-137 (2009).

23. McGibbon, E., Peter, E., Gallop, R. An institutional ethnography of nurses' stress. Qual Health Res. 20, (10), 1353-1378 (2010).

24. Orly, S., Rivka, B., Rivka, E., Dorit, S. E. Are cognitive-behavioral interventions effective in reducing occupational stress among nurses. Appl Nurs Res. 25, (3), 152-157 (2012).

25. Smith, S. A. Mindfulness-Based Stress Reduction: An Intervention to Enhance the Effectiveness of Nurses' Coping With Work-Related Stress. Int J Nurs Knowl. 25, (2), 119-130 (2014).

26. Bazarko, D., Cate, R. A., Azocar, F., Kreitzer, M. J. The Impact of an Innovative Mindfulness-Based Stress Reduction Program on the Health and Well-Being of Nurses Employed in a Corporate Setting. J Workplace Behav Health. 28, (2), 107-133 (2013).

27. Foureur, M., Besley, K., Burton, G., Yu, N., Crisp, J. Enhancing the resilience of nurses and midwives: pilot of a mindfulness-based program for increased health, sense of coherence and decreased depression, anxiety and stress. Contemp Nurse. 45, (1), 114-125 (2013).

28. Moody, K., et al. Helping the helpers: mindfulness training for burnout in pediatric oncology--a pilot program. $J$ Pediatr Oncol Nurs. 30, (5), 275-284 (2013).

29. Jha, A. P., Krompinger, J., Baime, M. J. Mindfulness training modifies subsystems of attention. Cogn Affect Behav Neurosci. 7, (2), 109-119 (2007).

30. Jha, A. P., Stanley, E. A., Kiyonaga, A., Wong, L., Gelfand, L. Examining the protective effects of mindfulness training on working memory capacity and affective experience. Emotion. 10, (1), 54-64 (2010).

31. Hölzel, B. K. Mindfulness practice leads to increases in regional brain gray matter density. Psychiatry Res. 191, (1), 36-43 (2011).

32. Hölzel, B. K. Stress reduction correlates with structural changes in the amygdala. Soc Cogn Affect Neurosci. 5, (1), 11-17 (2010).

33. Greeson, J. M. Mindfulness Research Update: 2008. Complement Health Pract Rev. 14, (10), $10-18$ (2009).

34. Beddoe, A. E., Murphy, S. O. Does mindfulness decrease stress and foster empathy among nursing students. J Nurs Educ. 43, (7), 305-312 (2004).

35. McCraty, R., Atkinson, M., Tomasino, D. Impact of a workplace stress reduction program on blood pressure and emotional health in hypertensive employees. J Altern Complement Med. 9, (3), 355-369 (2003).

36. Stone, P. W., et al. Nurse working conditions, organizational climate, and intent to leave in ICUs: an instrumental variable approach. Health Serv Res. 42, (3 pt. 1), 1085-1104 (2007).

37. McHugh, M. D., Kutney-Lee, A., Cimiotti, J. P., Sloane, D. M., Aiken, L. H. Nurses' widespread job dissatisfaction, burnout, and frustration with health benefits signal problems for patient care. Health Aff (Millwood). 30, (2), 202-210 (2011).

38. Klatt, M. D., Buckworth, J., Malarkey, W. B. Effects of low-dose mindfulness-based stress reduction (MBSR-ld) on working adults. Health Educ Behav. 36, (3), 601-614 (2009).

39. Carmody, J., Baer, R. A. How long does a mindfulness-based stress reduction program need to be? A review of class contact hours and effect sizes for psychological distress. J Clin Psychol. 65, (6), 627-638 (2009).

40. Bishop, S. R., et al. Mindfulness: A proposed operational definition. Clinical psychology: Science and practice. 11, (3), $230-241$ (2004).

41. Connor, K. M., Davidson, J. R. Development of a new resilience scale: The Connor-Davidson resilience scale (CD-RISC). Depression and Anxiety. 18, (2), 76-82 (2003).

42. Campbell-Sills, L., Murray, B. Psychometric analysis and refinement of the connor-davidson resilience scale (CD-RISC): Validation of a 10item measure of resilience. Trauma Stress. 20, (6), 1019-1028 (2007).

43. Schaufeli, W. B., Bakker, A. B., Salnova, M. The measurement of work engagement with a short questionnaire. A cross-national study. Edu and Psych Measur. 66, (4), 701-716 (2006). 